\title{
Die Umstellung unserer Zeitschrift auf Open Access und Komplett- Digitalisierung
}

\author{
Caroline Villiger, Pädagogische Hochschule Bern \\ Danièle Périsset, Haute école pédagogique du Valais et Université de Genève \\ Stefano Losa, Scuola universitaria professionale della Svizzera italiana SUPSI, \\ Locarno
}

«Wer nicht mit der Zeit geht, geht mit der Zeit». Die Redaktion der Schweizerischen Zeitschrift für Bildungswissenschaften hat sich zusammen mit dem Vorstand der Schweizerischen Gesellschaft für Bildungswissenschaften (SBGF) entschieden, mit der Zeit zu gehen, um nicht mit der Zeit zu gehen...

Seit bald zwei Jahrzehnten ist ein Wandel des Verhältnisses zwischen Wissenschaft und Gesellschaft zu beobachten. Es ist die Rede von einer «Vergesellschaftung» der Wissenschaft (Weingart, 2011). Die Wissensgesellschaft ist das Produkt einer massiven Bildungsrevolution, die aufgrund der Digitalisierung und dem damit einhergehenden vereinfachten Zugang zu Wissen noch beschleunigt worden ist. Diese Entwicklungen verleihen der Wissenschaft einen neuen Status innerhalb der Gesellschaft: Sie repräsentiert nicht länger den Elfenbeinturm als Ort, wo Wissen geschaffen wird, das nur einer ausgewählten Gesellschaftsgruppe zugänglich ist. Von Seiten der Politik und Gesellschaft zeigen sich zudem wachsende Erwartungen an die Sichtbarkeit und Nützlichkeit der mit öffentlichen Geldern finanzierten Wissenschaft (Stichwort Legitimationsdruck). Dies führt zu neuen Governancestrukturen, bei denen wissenschaftsfremde Anforderungen zunehmend an Bedeutung gewinnen (vgl. Grande, Jansen, Jarren, Rip, Schimank \& Weingart, 2013).

Diese Umwälzungen bleiben nicht ohne Auswirkungen auf das Publikationswesen. Die Forderung nach öffentlich zugänglichen Publikationen hat mittlerweile weltweit Einzug gehalten. Sie stützt sich hauptsächlich auf zwei Missstände, nämlich 1) auf die unangemessen hohen Preise von Zeitschriftenabonnements der dominierenden Wissenschaftsverlage und 2) auf die Tatsache, dass Publikationen nicht universell zugänglich sind und deren Zugang stark vom Budget wissenschaftlicher Einrichtungen abhängt. Dem wird gegenüber gestellt, dass Wissen bzw. Forschungsergebnisse, deren Entstehung weitgehend mit öffentlichen Geldern finanziert wurde, ein öffentliches Gut sei und daher zeitnah, digital, uneingeschränkt und kostenlos für alle zugänglich sein sollte (BOAI, 2002). 
Auch in der Schweiz wird die Debatte um den offenen Zugang zu wissenschaftlichen Erkenntnissen mittlerweile seit gut 10 Jahren geführt und Stellungnahmen werden von wissenschaftsrelevanten Gremien formuliert. Der Schweizerische Nationalfonds zur Förderung der wissenschaftlichen Forschung (SNF) hatte bereits 2006 eine entsprechende Positionierung unterzeichnet, die sich hinter die Forderungen der Open-Access-Initiative stellt (Hirschmann \& Verdicchio, 2017). Swissuniversities haben schliesslich 2017 eine Nationale Open-Access-Strategie für die Schweiz verabschiedet, worin als Vision festgehalten ist, dass bis zum Jahr 2024 alle mit öffentlichen Geldern finanzierten wissenschaftlichen Publikationen öffentlich zugänglich (Open Access oder abgekürzt OA) sein sollten (Swissuniversities, 2017). Auch die Schweizerische Akademie der Geistes- und Sozialwissenschaften (SAGW) zieht am selben Strick und fordert, dass die von ihr finanziell unterstützten Zeitschriften ab 2020 nur noch im Open Access erscheinen (SAGW, 2016).

Die Forschenden ihrerseits sind ausdrücklich dazu aufgefordert, sich für Open Access und im weiteren Sinne für Open Science, eine offene Wissenschaftskultur des Austauschs und der Zusammenarbeit unter Forschenden, zu engagieren.

Die SZBW gehört nicht zu den umstrittenen Hybrid-Zeitschriften, die sich sowohl über hohe Abonnementspreise als auch über Autorengebühren für Open Access-Beiträge finanzieren - streng genommen war sie bereits zuvor eine Zeitschrift mit «Green Open Access». Nach einer Sperrfrist von 12 Monaten waren die publizierten Beiträge im Internet frei zugänglich. Was also ist neu? Ab 2019 wird die Schweizerische Zeitschrift für Bildungswissenschaften als Open Access-Zeitschrift ohne Sperrfrist erscheinen. Dies bedeutet, dass die publizierten Beiträge nach Erscheinen der Nummer sofort digital zugänglich sind. Die Mitglieder der SGBF werden über die Herausgabe einer neuen Nummer informiert und können sie im Internet einsehen, einzelne Beiträge herunterladen oder ausdrucken. Gleichzeitig erfährt die Zeitschrift mit der Migration in ein Zeitschriftenverwaltungssystem (Open Journal Systems - OJS) eine erhöhte Digitalisierung (z.B. Vergabe von doi-Nummern, Eintrag im Directory of Open Access Journals - DOAJ), was die Sichtbarkeit und Auffindbarkeit der Zeitschrift und ihrer publizierten Beiträge erhöht. Weiter sind die Einreichungs-, Begutachtungs- und Publikationsprozesse aufgrund der Digitalisierung sowohl für die Autorinnen und Autoren als auch für die Redaktion einfacher und übersichtlicher gestaltet und der Status jedes eingereichten Beitrags ist jederzeit über das Internetportal abrufbar. Die Zeitschrift wird von BOP-Serials (Bern Open Publishing), einer Dienstleistung der Universitätsbibliothek Bern, gehostet, die den technischen Betrieb des Systems sichergestellt. Darüber hinaus sind sämtliche Ausgaben der SZBW (inklusive Vorgängerzeitschrift "Bildungsforschung und Bildungspraxis», seit 1979!) auch retrodigitalisiert auf e-periodica, der Schweizer Plattform für digitalisierte Zeitschriften der ETH-Bibliothek Zürich, zugänglich. 
Insgesamt steigt die Attraktivität der Zeitschrift für Forschende, die, wie bereits erwähnt, in Zukunft noch expliziter dazu angehalten sind, Open Access zu publizieren. $\mathrm{Zu}$ bedauern ist auf der anderen Seite die Tatsache, dass unsere Zeitschriftensammlung im Büchergestell und in diversen Bibliotheken im Inund Ausland nun abrupt abbricht. All diejenigen Leserinnen und Leser, die sich jeweils über die postalische Zustellung der Zeitschrift gefreut und sich die neueste Ausgabe für die nächste Zugreise eingesteckt hatten, sind aufgefordert umzudenken und neue Gewohnheiten zu entwickeln. Kritisch kann an dieser Stelle angemahnt werden, dass einmal mehr der taktile Lesegenuss, gezeichnet von der unbegrenzten Möglichkeit des Weiter- und Zurückblätterns, des nicht dringend zweckdienlichen Lesens, weil ganz zufällig etwas Unbekanntes unsere Aufmerksamkeit auf sich zieht, schliesslich der trendbehafteten Zweckorientierung (durch die digitalisierten und in Einzelartikel segregierten Ausgaben akzentuiert) und Flüchtigkeit (ohne Hinterlassen materieller Spuren) weicht.

Was wir bei diesen Umstellungen im Auge behalten: Trotz Digitalisierung ist es der Redaktion wichtig, den persönlichen Kontakt mit Gutachtenden, Autorinnen und Autoren wie bis anhin zu pflegen. Weiter soll die Umstellung auf Komplett-Digitalisierung in keiner Weise die Qualität der Zeitschrift verringern. Unseren Auftrag, für eine Qualitätskontrolle im Wissenschaftsbetrieb zu sorgen, nehmen wir weiterhin uneingeschränkt wahr, zumal die SZBW aufgrund ihres Finanzierungsmodells sich nicht gezwungen sieht, aus wirtschaftlichen Gründen mehr (schlechte) Artikel anzunehmen.

Die Herstellung der Zeitschrift ist trotz wegfallendem Druck mit Kosten verbunden. Der Begutachtungsprozess und die editorische Vorbereitung der Beiträge erfordern nach wie vor dieselbe Arbeit. Neu fallen Kosten für das Hosting der Zeitschrift an. Die SGBF ist auf die Unterstützung der Schweizerischen Akademie der Geistes- und Sozialwissenschaften (SAGW) angewiesen, aber diese Beiträge alleine reichen nicht. Deshalb bedanken wir uns bei den SGBF-Mitgliedern, denen, so hoffen wir, an «ihrem» Publikationsorgan gelegen ist und es weiterhin mit ihrem Mitgliederbeitrag unterstützen.

Diese erste komplett-digitalisierte Ausgabe 1/2019 ist eine Varia-Nummer. Die 11 publizierten Artikel stammen wie immer aus unterschiedlichen Sprachregionen und Ländern (Schweiz, Frankreich, Belgien und Deutschland) und behandeln eine Vielfalt von bildungswissenschaftlichen Fragestellungen, auf die wir hier nicht näher eingehen. Sieben Rezensionen geben jeweils kurze, aber gehaltvolle Einblicke in neuere Buchpublikationen.

Wir wünschen Ihnen eine angenehme Lektüre und anregende Impulse für Ihre eigenen Arbeiten! 


\section{Literatur}

BOAI (2002). Budapest Open Access Initiative. Zugriff am 18.02.2019 https://www.budapestopenaccessinitiative.org/read

Grande, E., Jansen, D., Jarren, O., Rip, A., Schimank, U. \& Weingart, P. (2013, Hrsg.). Neue Governance der Wissenschaft. Reoganisation - externe Anforderungen-Medialisierung. Bielefeld: transcript.

Hirschmann, B. \& Verdicchio, D. (2017). Open Access in der Schweiz. In K. Söllner \& B. Mittermaier (Hrsg.), Praxishandbuch Open Access, S. 215-222. Berlin/Boston: De Gruyter.

SAGW (2016). Open-Access-Strategie der Schweizerischen Akademie der Geistes- und Sozialwissenschaften. Zugriff am 18.02.2019 http://www.sagw.ch/dms/sagw/open_access/OpenAccess_Strategie_def_d.

Swissuniversities (2017). Nationale Open-Access-Strategie für die Schweiz. Zugriff am 18.02.2019 https://www.swissuniversities.ch/fileadmin/swissuniversities/Dokumente/ Hochschulpolitik/Open_Access/Open_Access_strategy_final_DE.pdf

Weingart, P. (2011, 3. Aufl.). Die Stunde der Wabrheit? Zum Verhältnis der Wissenschaft zur Politik, Wirtschaft und Medien in der Wissensgesellschaft. Weilerswist: Velbrück Wissenschaft. 